\title{
Effect of the Directional Characteristic of the Waves Sensor ME36 on the Measurement of the Speech Transmission Index STIPA and Comprehensibility CIS
}

\author{
René Drtina $^{1, a}$, Pavel Fejfar ${ }^{1, b}$, Josef Sedivy ${ }^{1, c}$, Oldrich Turecek ${ }^{1, d}$ \\ 1,2,3,4 University of Hradec Kralove, Faculty of education, Department of technical subject \\ Rokitanskeho 62, 50003 Hradec Kralove, Czech Republic \\ arene.drtina@uhk.cz, ${ }^{\mathrm{b}}$ pavel.fejfar@uhk.cz, ${ }^{\mathrm{a} j o s e f . s e d i v y @ u h k . c z,}{ }^{\mathrm{d}}$ oldrich turecek@uhk.cz
}

Keywords: speech transmission index, clarity, sensor, directional characteristic, pressure microphone, hypercardioid microphone wave sensor.

Abstract: The acoustic transmission channel has a unique place in the information transfer in all spheres of life. The dominant position acoustic communication today keeps prsonálním process information transfer. Yet achievable intelligibility in classrooms, lecture halls and a congress does not pay close attention. The existing research results show that measurement of the speech transmission index Stipe can not agree with logatomickými tests for evaluating the transmission properties of instructional space and the measurement results are dependent on the characteristics of the directional measurement microphone. Article presents preliminary results of comparative research on the influence directional characteristics of wave sensor, which simulates binaural hearing mono signal to the measured values STIPA and CIS.

\section{Introduction}

The research plan, which builds on previous research projects speech intelligibility in classrooms and lecture halls, and comparing the measurement methods, the output parameter is a single number, clearly demonstrated the influence of directional characteristics of the used sensors to the measured value. Research has proven that a pair of Sennheiser transducers hyperkardiodních MD441U simulates conditions far better binaural hearing in healthy humans [1]. Another comparative research has focused on simulating binaural hearing using a short wave transmitter type "short gun".

\section{Ripple sensor ME36}

Wave (or also line) microphones are among the directional sensor. Cylinder horn connected to a pressure sensor has almost the entire length of the slits or openings are covered with an acoustic resistance such that all signals entering the ear canal reached a pressure sensor with the same intensity [2,3]. Principal solutions wave sensor is shown on figure 1. Adjusting the damping, it is possible to influence the properties of the auditory canal such that its effective length decreases with decreasing wavelength of the incident sound wave (effective length decreases with increasing frequency). Ideally, the directional characteristics of sensor frequency independent [4].

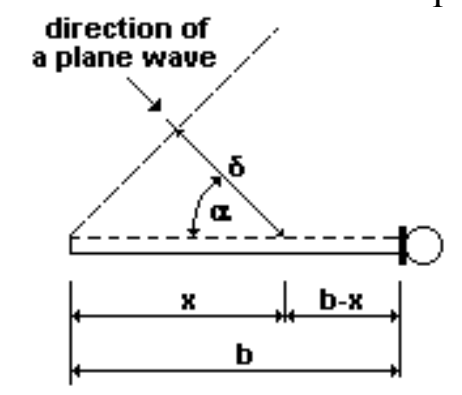

Fig.1 Principle solutions of wave sensors 
Suppose that a plane sound wave propagating at the speed c, which turns at an angle $\alpha$ from the acoustic axis of the transducer, enter the distance $\mathrm{x}$ into the ear canal with a delay $\Delta t$, which is proportional to the path difference

$\delta=x \cos \alpha \quad(1)$

and $\Delta t=\frac{\delta}{\mathrm{c}}=\frac{x}{\mathrm{c}} \cos \alpha$

Phase delay signal of frequency $f$ at a distance $x$ is

$\varphi=2 \pi f \frac{x}{c}(1-\cos \alpha)$

The aggregate level of both signals at a distance $x$ will then be

$A_{\mathrm{M}}=2 A_{0} \sin \pi f \frac{x}{\mathrm{c}}(1+\cos \alpha) \cdot \cos \pi f \frac{x}{\mathrm{c}}(1-\cos \alpha)$

For the directional characteristics of the standard solved wave sensors can derive an equation standardized directional function of the form

$$
A=\frac{\sin \pi \frac{\mathrm{b}}{\lambda}(1-\cos \alpha)}{\pi \frac{\mathrm{b}}{\lambda}(1-\cos \alpha)}
$$

where after substituting for $\lambda=x / f$ we get

$$
A=\frac{\sin \pi \frac{\mathrm{b} f}{\mathrm{c}}(1-\cos \alpha)}{\pi \frac{\mathrm{b} f}{c}(1-\cos \alpha)}
$$

Examples theoretical course of direction of the wave functions of the sensor according to the equation (6) with an active length of $8,5 \mathrm{~cm}$ are shown on figure 2.
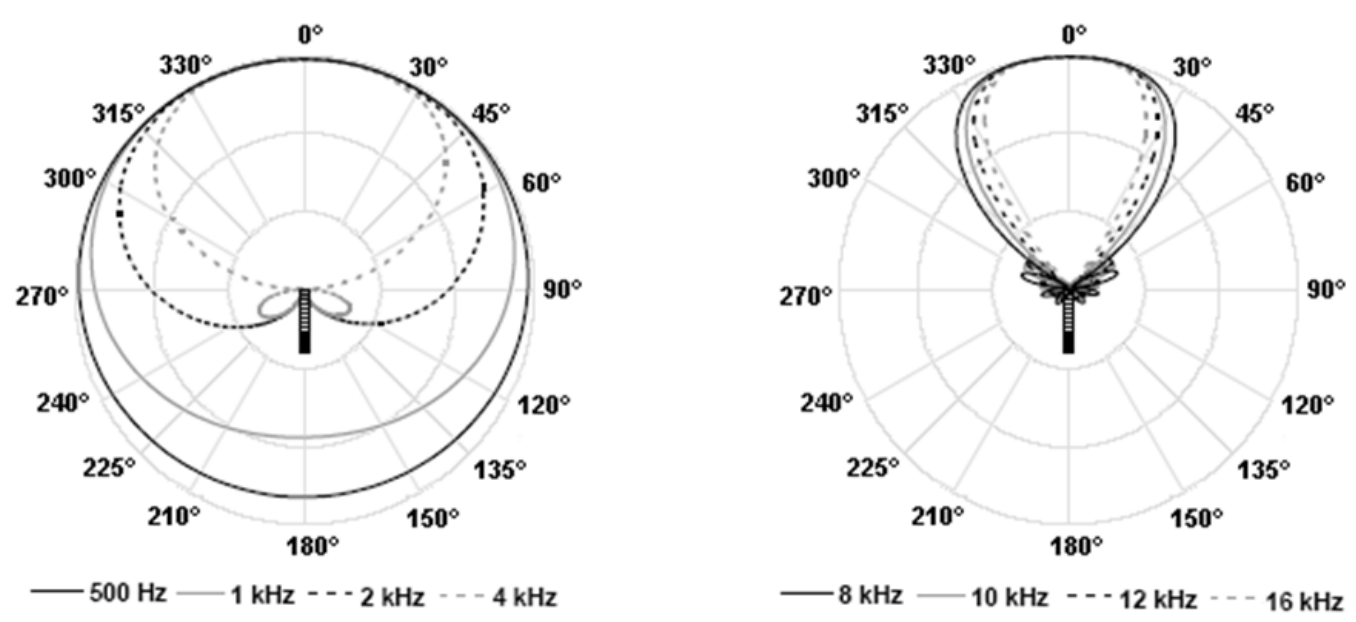

Fig.2 Theoretical courses directional wave sensor function of length $8.5 \mathrm{~cm}$

Wave sensor manufacturer Sennheiser ME36 classified as a hypercardioid microphone with a narrow directional characteristics. Its dimensions are $8,2 \times 96 \mathrm{~mm}$, active length $85 \mathrm{~mm}$, as called. Short gun is among the top products of its type. Measurement of directional characteristics are realized as sensors MD441U multitone signal in an anechoic chamber with a reference microphone Brüel \& Kjær analyzer and BK Pulse 3560C. The measurement results are on figure 3. Like all acoustic emitters and sensors, creating even wave sensor side lobes. When comparing the directional characteristics (fig. 3) and a directional function (fig. 2) it is clear that the side lobes are 
created especially for higher frequencies. Due to standardization during the directional functions but not in the charts as sharp as when using logaritmic scale with directional characteristics.
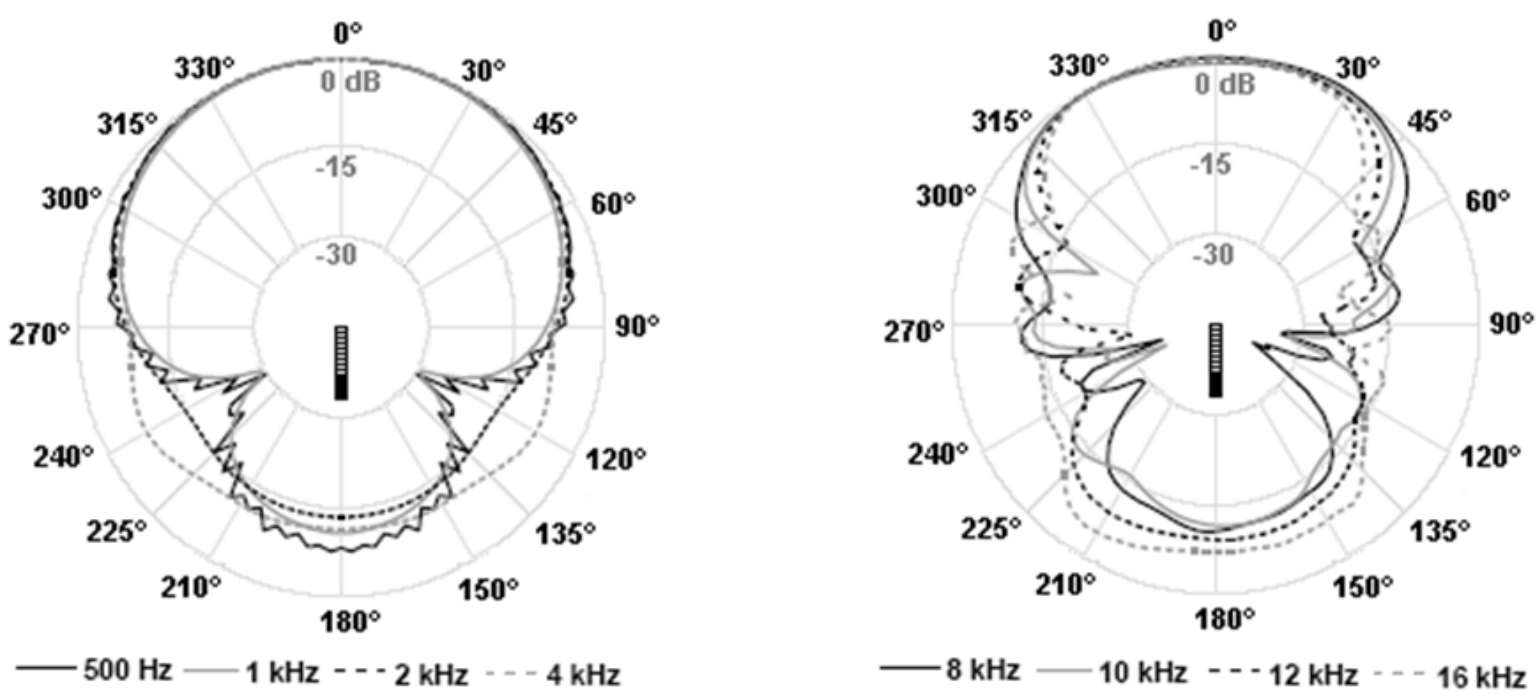

Fig.3 Measurements directional characteristics of the wave transmitter ME36

The evaluation of the directional characteristics that the suppression of the rear signal is at -8 to $14 \mathrm{~dB}$, the side signals are then suppressed at -9 to $-21 \mathrm{~dB}$ (neglecting null directions).

\section{Results of a comparative index measurements speech transmission}

Effect of directional characteristics of sensors for measuring the index of speech transmission STIPA and clarity CIS we tested in space with a reverberation time of 1,5 $\mathrm{s}$ at a distance of 1-6 m from the reference emitter NTi TalkBox. On Fig. 4 the values STIPA CIS and measured wave sensor ME36 compared to the values measured pressure microphone NTi M2210 with balls characteristic.

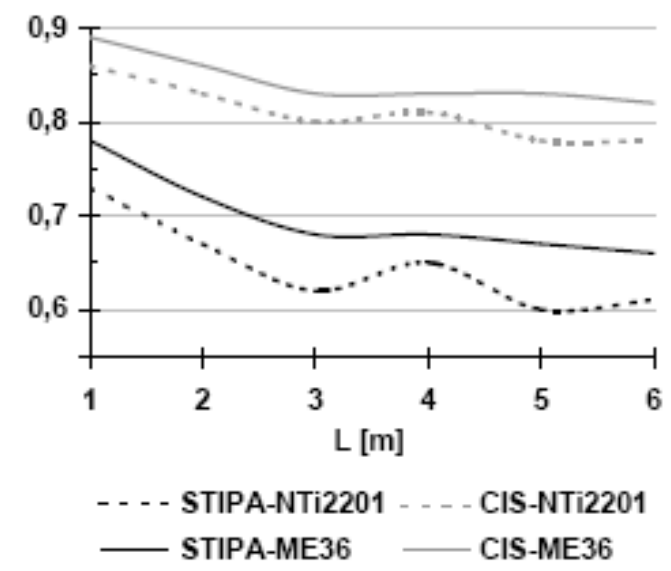

Fig.4 Comparison of measured values STIPA and CIS

The results of the measurements, we can infer the assumption that the directional characteristic wave sensor has a significant influence on the measurement results, both in free field and in diffuse field. From the course of the measured values we show that the results obtained by wave sensors are less sensitive to local variations. This is seen, for example at a distance of $4 \mathrm{~m}$, which corresponds approximately to the geometric center of the space. 

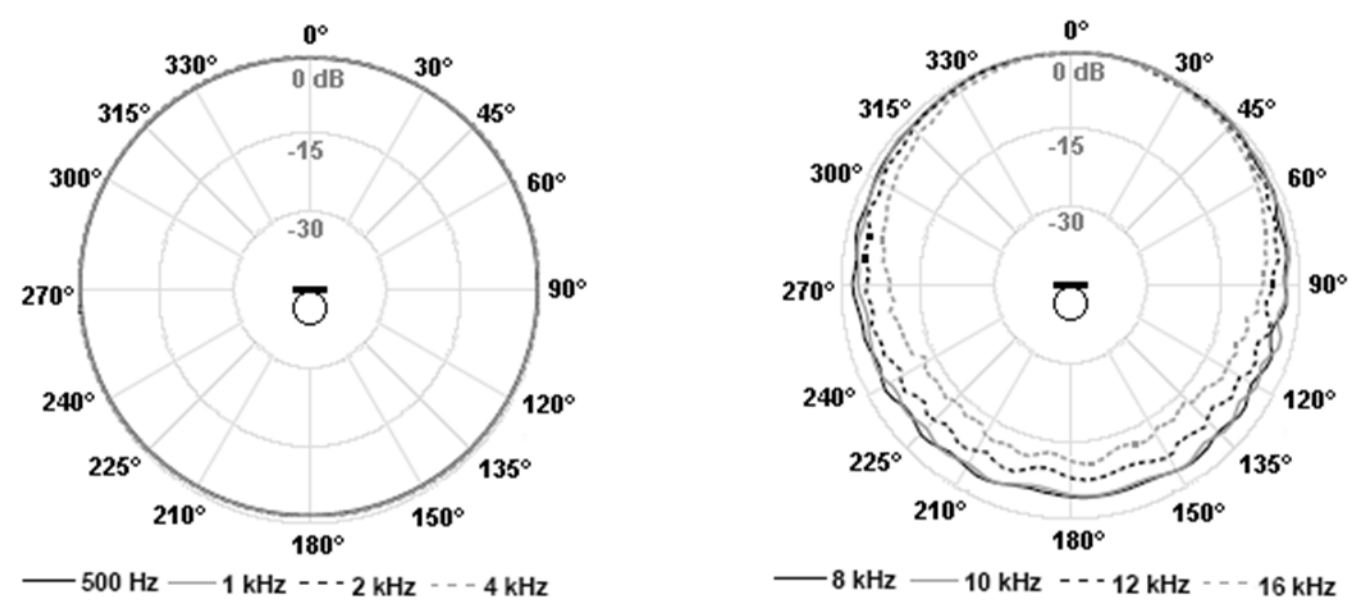

Fig.5 directional measurement microphone characteristics NTi M2210

While approaching wave sensor directional characteristics of binaural hearing mono signal, pressure microphone fails to comply with the direction of the incoming sound waves and largely represents one side of a hearing man. Like a pair of sensors and sensor suppresses wave signals coming from the rear and side sensors.

\section{Conclusion}

Partial results of research have shown that the influence of the directional characteristics of the sensor to the measured value speech transmission index STIPA and derived values of clarity, the CIS is significant. Another direction of research activity will therefore be directed towards finding suitable methods and comparative audioprocesingu possible to validate the measured values with the results logatomic tests.

In the future we will need to establish the method for the calculation of the correction of the measured values with respect to a transmitted frequency band. STIPA method incorrectly indicates a worsening of speech transmission index damping at low frequencies, which are on their own intelligibility of speech virtually zero share. In practice, it is clearly proven that just suppressing low frequencies of speech signal actually increases intelligibility in spaces with long reverberation time, since the sonic energy is restricted to long doznívajích tones.

\section{Acknowledgements}

The article was prepared with the support of specific research project SV 2133 PdF UHK dependence of the measured speech transmission index STIPA directional and frequency characteristics of acoustic receivers.

\section{References}

[1] D. Y. Huang, "A New Method to Measure the Absorption Coefficient Based on the Sound Pressure Delaying", Advanced Materials Research, Vols. 834-836, pp. 1156-1160, Oct. 2013

[2] P. Voborník, "Mini-Language for Effective Definition of the Color Gradients", Advanced Materials Research, Vols. 1030-1032, pp. 1882-1885, Sep. 2014

[3] R. Nemec, S. Hubalovsky, "Development of System SMPSL for Bit Communication", Applied Mechanics and Materials, Vols. 475-476, pp. 871-874, Dec. 2013

[4] E. Fumiaki, T. Toshiaki, K. Tanaka, "Development of a Heart Rate Measurement Method Using Body Conduction Sound Sensor", Applied Mechanics and Materials, Vol. 103, pp. 616621, Sep. 2011 